\title{
Papillary Glioneuronal Tumor
}

National Cancer Institute

\section{Source}

National Cancer Institute. Papillary Glioneuronal Tumor. NCI Thesaurus. Code C92554.

A WHO grade I, indolent and relatively circumscribed brain tumor. Morphologically it is characterized by the presence of astrocytes that line vascular and hyalinized pseudopapillae. In between the pseudopapillae aggregates of neurocytes, large neurons, and gang lioid cells are present. 